\title{
The Effect of Corrosion on the Natural Frequencies of Beams
}

\author{
Steliana Stanciu ${ }^{1}$, Dorin Simoiu ${ }^{1}$, Ion Crâştiu ${ }^{1}$, Sorin Deac ${ }^{1}$, Eduard Nicuşor Oanţă ${ }^{1}$ and \\ Liviu B ereteu ${ }^{1 *}$, \\ ${ }^{1}$ Politechnica U niversity Timisoara, M echanics and M aterials Strength Department, 300222 B -dul \\ M ihai V iteazul, no.1, Timisoara Romania
}

\begin{abstract}
Corrosion is a natural process of degradation of the mechanical properties of some materials like: metals, ceramics or polymers. The metals and their alloys corrode only because of their presence in a damp environment, but the process is more powerful in some corrosive environments. Corrosion is a diffusion process by which some chemical bonds of the base material are broken, and therefore the oxides, bases, or salts are formed, resulting in a process of mass loss but also of weak interatomic bonding forces, that means a loss of stiffness. M ass distribution and stiffness in a mechanical structure determine the natural frequencies and shape of natural modes, that is, so-called modal parameters that characterize its dynamic behavior. The purpose of this paper is to determine the influence of simultaneous loss mass and loss stiffness on natural frequencies. A pplying the Finite Element Method and the Modal Analysis Module of the ANSYS software, the natural frequencies are obtained in numerical approach, which compares with the obtained analytical values, to confirm the validity of the proposed method.
\end{abstract}

\section{Introduction}

M echanical structures subjected to corrosion degradation can be affected both in safety and in service life. The corrosive defects generally lead to the modification of some mechanical characteristics, characteristics which, in terms of vibrations, are highlighted by forms of natural vibration modes and natural frequencies. The evaluation of large mass losses through corrosion and their influence on natural frequencies is analyzed in [1-2]. In analyzing the vibrations of some mechanical structures on beam-type models, even if considered to be one-dimensional, it should be taken into account that bending vibrations and torsional vibrations can be coupled. Therefore, in the analysis with finite elements these effects can lead to very large differences in their natural frequencies, higher than those given by the damage. The effects of corrosion and damping on natural frequencies of concrete steel beams are presented in paper [3]. In this paper it is shown that for a rectangular section beam, if the length is at least eight times the width and the width is at least eight times the thickness, then the bending vibrations in the two planes and the

* Corresponding author: liviu.bereteu@ upt.ro 
torsional vibrations are decoupled. The effects on the natural frequencies given by the defect depth, its position and its width are analyzed.

\section{Vibration analysis of beam}

In the dynamic analysis of a continuous and homogeneous one-dimensional structure, that is, with a much larger dimension than the others, bending vibrations coupled with torsion can occur. B eams, trees, axles, and axes are in the category of these structures. If the cross section has two axis of symmetry, then the center of gravity and the center of shear will be found at the intersection of these axes. B eams that have a rectangular section fall into this category, and the differential equations that govern their vibrations are separated into two equations of bending vibrations in the OXY and OXZ planes, and an equation of torsional vibrations.

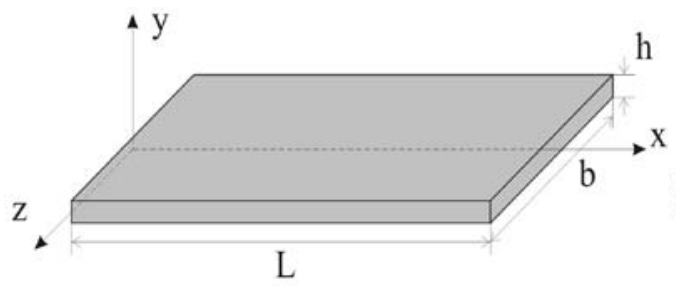

Fig. 1. B eam model

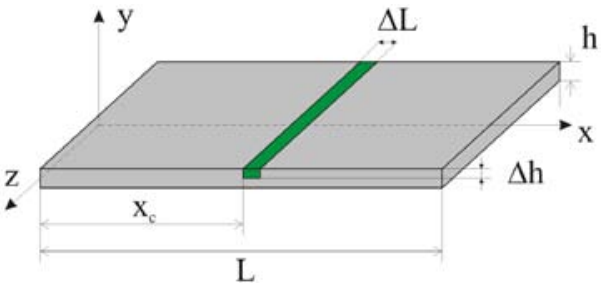

Fig.2. Phisical corroded beam model $\stackrel{0}{.}$.

A free-free slender elastic beam with $L$ length, $b$ width and $h$ heigth is considered for vibration analysis. The vibrational bending motion in the OXY plane of the uncorroded beam is governed by the following differential equation

$$
E I_{z} \frac{\partial^{4} y(x, t)}{\partial x^{4}}+\rho A \frac{\partial^{2} y(x, t)}{\partial t^{2}}=0,
$$

where $y(x, t)$ denoted the displacement in the $O X Y$ plane, $E$ is the longitudinal elastic modulus, $I_{Z}$ is the moment of inertia of the cross section with respect to the $O Z$ axis, $\rho$ is the density of the material, and $A$ is the area of the cross section of the beam.

For the analytical solving of the differential equation of bending vibrations was considered the method of separating the variables and each point of the bar has a harmonic vibratory motion

$$
y(x, t)=Y(x) \sin \omega t
$$

A fter separating the variables, the functions that give the shape of natural vibration modes are obtained by solving the differential equation

$$
\frac{\partial^{4} Y(x)}{\partial x^{4}}-\lambda^{4} Y(x)=0
$$

where $\lambda$ is the value obtained from the expression

$$
\lambda^{4}-\omega^{2} \frac{\rho L}{E I_{z}}=0
$$

From the boundary conditions imposed for the beam having the free ends it is obtain the characteristic equation, whose solutions are the eigenvalues of the problem. Noting with $X=\lambda L$, the characteristic equation is

$$
1-\cosh X \cos X=0 \text {. }
$$


It can be shown that with the exception of the first rigid motion of the body in the OXY plane, the solutions of equation (5) are

$$
X_{i}=\frac{2 i+1}{2} \pi, i=1,2,3 \ldots \ldots
$$

$B$ ased on equations (4) and (6) the natural frequencies of the bending vibrations of the beam in the OXY plane are

$$
f_{i}=\frac{X_{i}^{2}}{2 \pi L^{2}} \sqrt{\frac{E I_{z}}{\rho A}}=\frac{(2 i+1)^{2} \pi}{8 L^{2}} \sqrt{\frac{E I_{z}}{\rho A}}, i=1,2,3 \ldots .
$$

For the bending vibrations of the bar in the $O X Z$ plane we obtain a differential equation of the same form as equation ( 1 )

$$
E I_{y} \frac{\partial^{4} z(x, t)}{\partial x^{4}}+\rho A \frac{\partial^{2} z(x, t)}{\partial t^{2}}=0
$$

where $z(x, t)$ denoted the displacement in the $O X Z$ plane, $E$ is the Iongitudinal elastic modulus, $I_{y}$ is the moment of inertia of the cross section with respect to the OY axis, $\rho$ is the density of the material, and $A$ is the area of the cross section of the beam.

Following the same steps to solve the differential equation of the bending vibrations of the beam in the $O X Z$ plane as in the previous case, it is obtain the natural frequencies given by the relations:

$$
f_{j}=\frac{X_{j}^{2}}{2 \pi L^{2}} \sqrt{\frac{E I_{y}}{\rho A}}=\frac{(2 j+1)^{2} \pi}{8 L^{2}} \sqrt{\frac{E I_{y}}{\rho A}}, j=1,2,3 \ldots .
$$

In the case of a rectangular cross-section bar, it has two axes of symmetry, therefore the differential equation of the torsional vibrations relative to the $O X$ axis is decoupled from the equations of the bending vibrations in the two planes and has the form (10) where: $\varphi(t)$ is the torsional angle of the cross-section, $G$ is the shear modulus, $\mathrm{I}_{\mathrm{T}}$ is the equivalent moment of inertia of cross-section due to torsion, also known as the torsion constant, and Jo is the polar mechanical moment of inertia of the cross-section.

$$
-G I_{T} \frac{\partial^{2} \varphi(x, t)}{\partial x^{2}}+J_{O} \frac{\partial^{2} \varphi(x, t)}{\partial t^{2}}=0,
$$

It should be specified that the equival ent moment of inertia of cross-section due to torsion is equal to the moment of inertia polar only for circular sections. For a non-circular crosssection during the process of torsion the deformation of cross-sections, sometimes called warping deformation, is observed. For a rectangular cross-section, the torsion constant can be determined with formula (11), and the moment of mechanical inertia is given by the product between the material density $\rho$, and the polar inertia moment of the cross-section $I_{0}$ [4].

$$
I_{T}=\frac{b h^{3}}{3}\left[1-0.63 \frac{h}{b}+0.052\left(\frac{h}{b}\right)^{5}\right]
$$

Applying the method of separating the variables in the case of torsional vibrations, following a procedure similar to the bending vibrations, it is obtain the formula (12) for determining the natural torsional frequencies of the uncorroded beam.

$$
f_{k}=\frac{k}{2 L} \sqrt{\frac{G I_{T}}{\rho I_{O}}}, k=1,2,3 \ldots .
$$




\section{Finite element modeling of uncorroded and corroded beam}

The first step in the vibration analysis of the beam is to find its theoretically natural frequencies in the free-free boundary conditions. The modeled beam has rectangular cross section with length $L=250 \mathrm{~mm}$, width $b=40 \mathrm{~mm}$ and height $h=5 \mathrm{~mm}$.

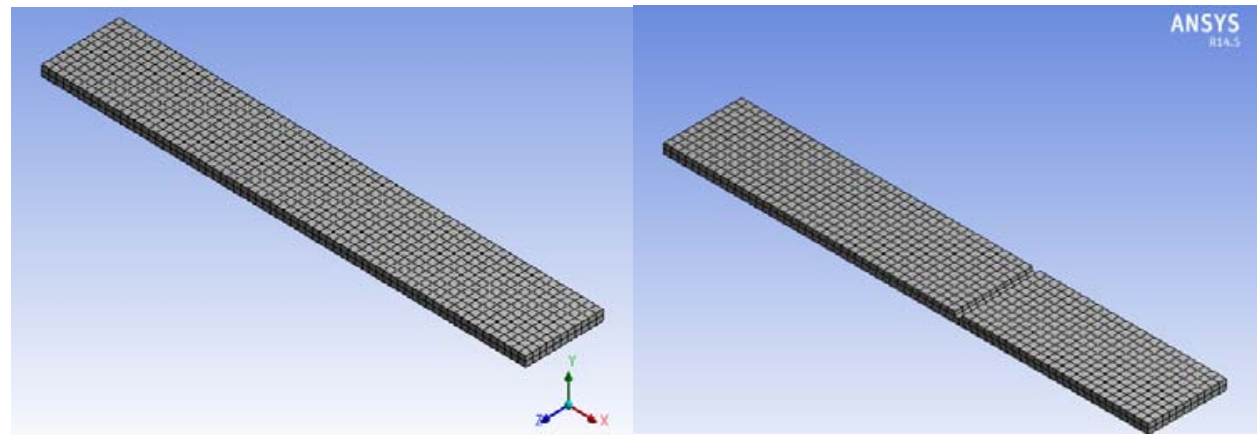

Fig. 3. Discretezed model of beam

Fig.4. Discretezed model of corroded beam with

In the theoretical analysis of the vibrations of a bar, it has been assumed that it has a section with symmetry axes and that it is much longer thin compared to the width so that the differential equations are decoupled. The numerical analysis performed on such a bar will be done in the free-border boundary conditions. For the numerical simulation of the dynamic behavior of the beam Finite Element A nalysis was performed using A NSY S 14.5 software. To construct a 3D model, hexahedral solid elements SOLID 186 with three degrees of freedom for each node were used.

Table 1.First three natural bending frequencies in OXY plane

\begin{tabular}{|c|c|c|c|}
\hline \multirow{2}{*}{$\begin{array}{l}\text { Mode } \\
\text { No }\end{array}$} & \multicolumn{2}{|c|}{ Frequency[Hz] } & \multirow[t]{2}{*}{ M ode shape } \\
\hline & Theory & FEM & \\
\hline 1 & 414.9 & 415 & 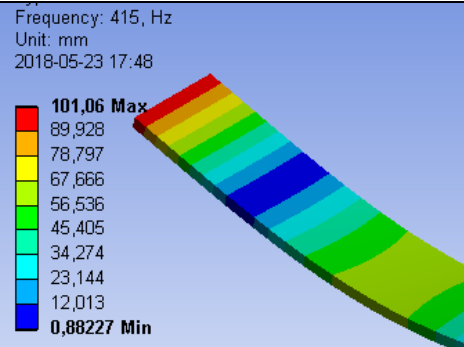 \\
\hline 2 & 1144 & 1144 & 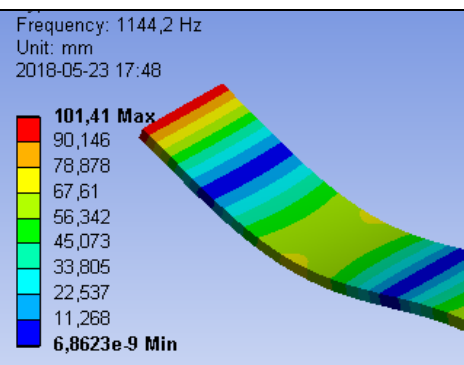 \\
\hline
\end{tabular}




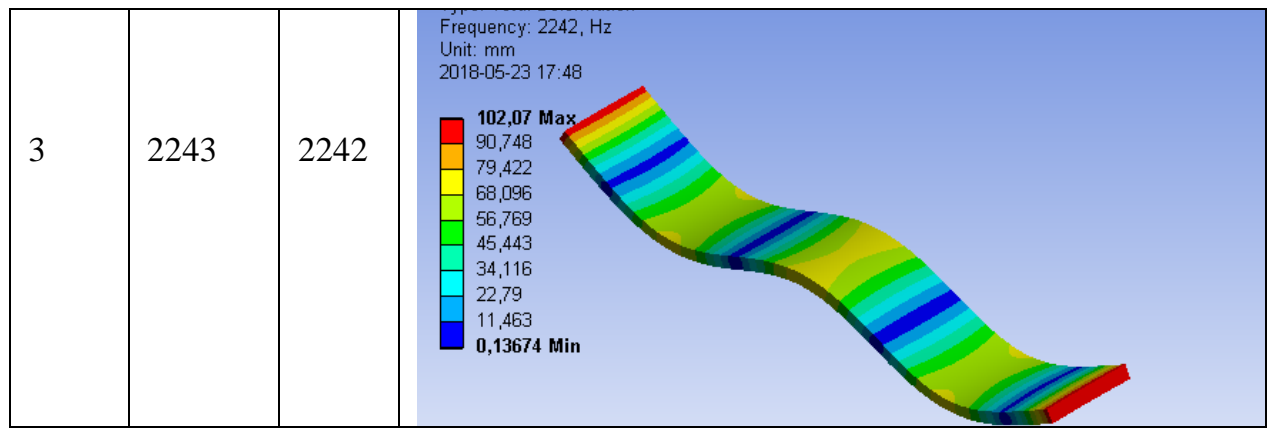

The first three modal shapes and corresponding natural frequencies for the uncorroded beam along with the theoretical natural frequencies obtained on the basis of the equations (7), (9) and (12), are presented in the tables Table 1, Table 2 and Table 3, corresponding to the bending vibrations in the OXY plane, bending vibrations in the OXZ plane and torsional vibrations.

Table 2.First three natural bending frequencies in OX Z plane

\begin{tabular}{|c|c|c|c|}
\hline \multirow{2}{*}{$\begin{array}{l}\text { Mode } \\
\text { No }\end{array}$} & \multicolumn{2}{|c|}{ Frequency[Hz] } & \multirow{2}{*}{ M ode shape } \\
\hline & Theory & FEM & \\
\hline 1 & 3075 & 3060 & 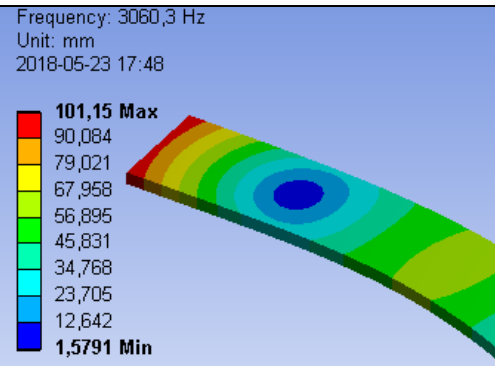 \\
\hline 2 & 7512 & 7489 & 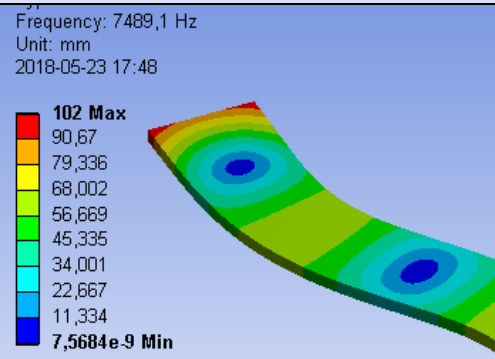 \\
\hline
\end{tabular}




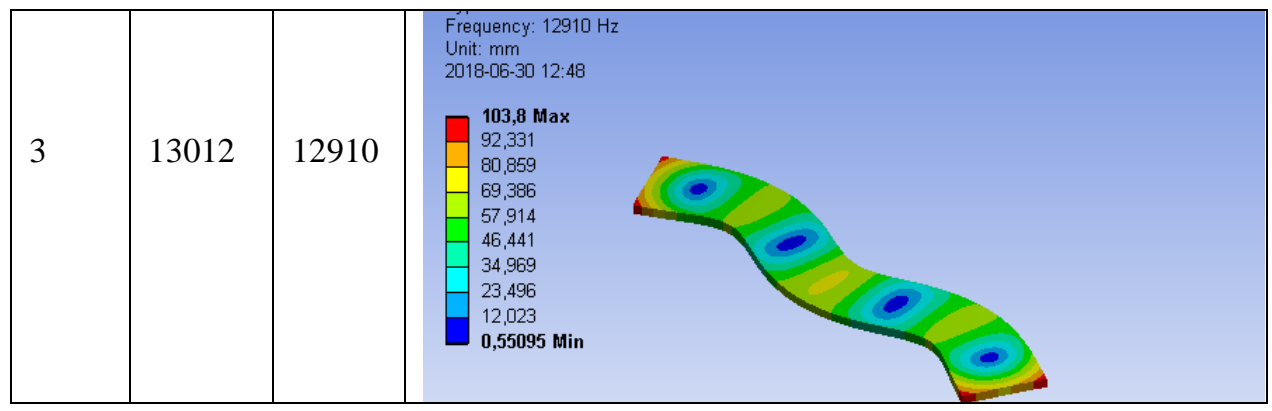

Table 3.First three natural torsion frequencies

\begin{tabular}{|c|c|c|c|}
\hline \multirow{2}{*}{$\begin{array}{l}\text { Mode } \\
\text { No }\end{array}$} & \multicolumn{2}{|c|}{ Frequency[Hz] } & \multirow{2}{*}{ M ode shape } \\
\hline & Theory & FEM & \\
\hline 1 & 1496 & 1499 & 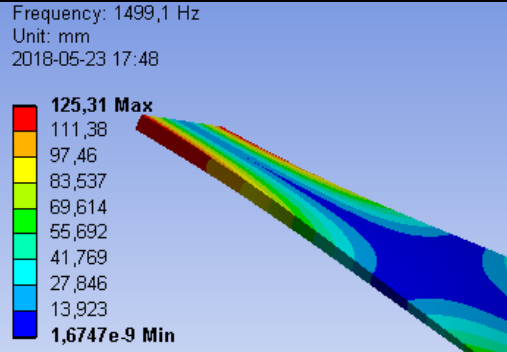 \\
\hline 2 & 3021 & 3046 & 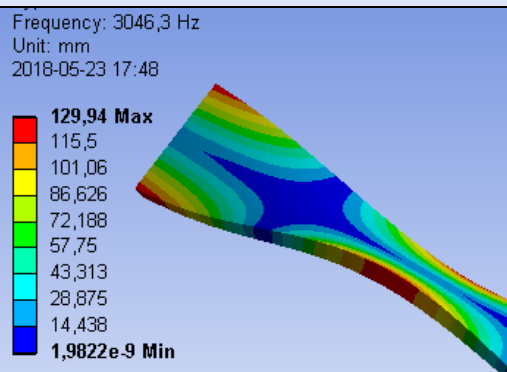 \\
\hline 3 & 4572 & 4687 & 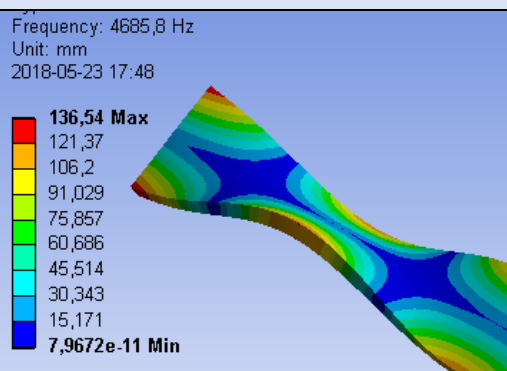 \\
\hline
\end{tabular}

Table 4 shows the variation of the frequencies of the first three bending modes of the beam, in the OXY plane, for a determined position of the corroded area at $X_{C}=150 \mathrm{~mm}$, having a corroded area width of $4 \mathrm{~mm}$, depending on different depths of penetration. 
Table 4.First three natural bending frequencies versus depth corrosion

\begin{tabular}{|l|l|l|l|l|l|}
\hline \multirow{2}{*}{ M ode No } & \multicolumn{5}{|l|}{ Frequency $[\mathrm{Hz}]$} \\
\cline { 2 - 6 } & uncorroded & $\Delta \mathrm{h}=0.5 \mathrm{~mm}$ & $\Delta \mathrm{h}=1 \mathrm{~mm}$ & $\Delta \mathrm{h}=1.5 \mathrm{~mm}$ & $\Delta \mathrm{h}=2 \mathrm{~mm}$ \\
\hline 1 & 415 & 411.4 & 405.3 & 398.5 & 386.3 \\
\hline 2 & 1144 & 1136.2 & 1129.3 & 1120 & 1104.4 \\
\hline 3 & 2242 & 2234.2 & 2233.6 & 2231 & 2223.7 \\
\hline
\end{tabular}

Table 5 presents the variation of the frequencies of the first three bending modes of the bar, in the OXY plane, for a determined position of the corroded area at Xc $=150 \mathrm{~mm}$, with the corrosion penetration depth $\Delta \mathrm{h}=1 \mathrm{~mm}$, depending on different widths of the corroded surface.

Table 5.First three natural bending frequencies versus width corrosion

\begin{tabular}{|l|l|l|l|l|l|}
\hline \multirow{2}{*}{ M ode No } & \multicolumn{5}{|l|}{ Frequency $[\mathrm{Hz}]$} \\
\cline { 2 - 6 } & uncorroded & $\Delta \mathrm{L}=4 \mathrm{~mm}$ & $\Delta \mathrm{L}=6 \mathrm{~mm}$ & $\Delta \mathrm{L}=8 \mathrm{~mm}$ & $\Delta \mathrm{L}=10 \mathrm{~mm}$ \\
\hline 1 & 415 & 405 & 403.6 & 400.9 & 399.2 \\
\hline 2 & 1144 & 1129.3 & 1126 & 1121 & 1117.4 \\
\hline 3 & 2242 & 2234.6 & 2234.3 & 2233.9 & 2234.3 \\
\hline
\end{tabular}

Table 6.First three natural bending frequencies versus position of corrosion

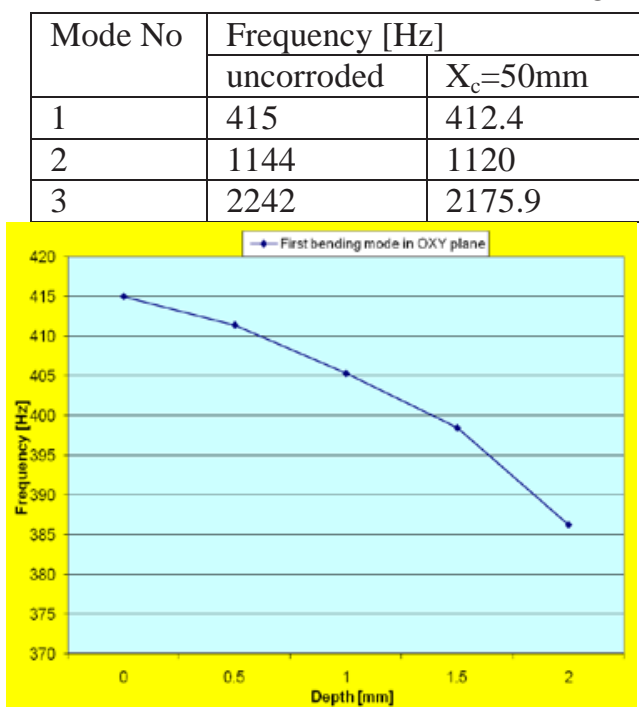

Fig. 5. First bending frequency versus depth

\begin{tabular}{|l|l|l|}
\hline$X_{c}=100 \mathrm{~mm}$ & $X_{c}=150 \mathrm{~mm}$ & $X_{c}=200 \mathrm{~mm}$ \\
\hline 403.88 & 405.3 & 412.3 \\
1130.5 & 1129.3 & 1122.6 \\
\hline 2226 & 2233.6 & 2174.3 \\
\hline
\end{tabular}
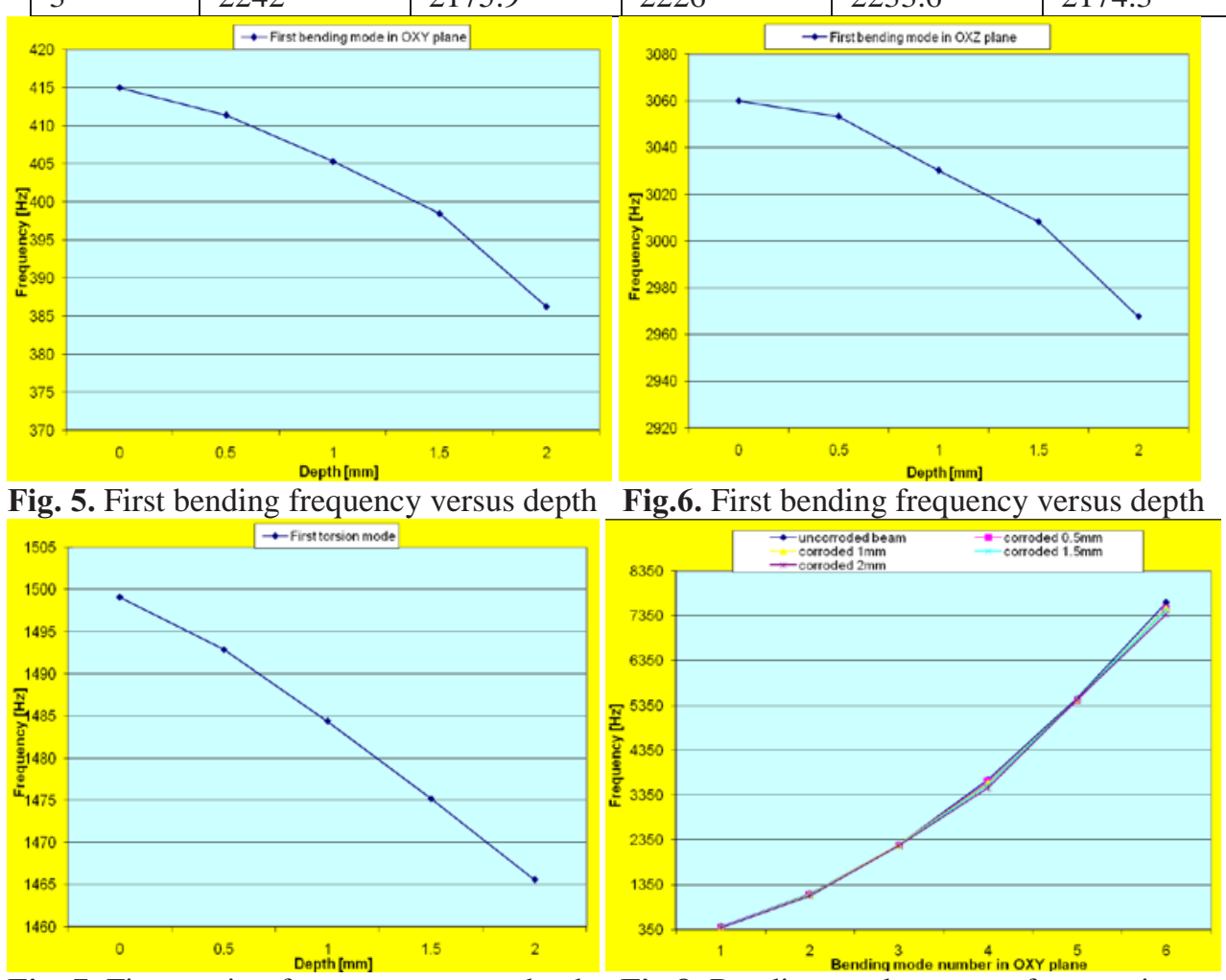

Fig. 7. First torsion frequency versus depth

Fig.8. B ending modes versus frequencies and depth corrosion 
Table 6 presents the variation of the frequencies of the first three bending modes of the bar, in the OXY plane, for the same pentetration $\Delta \mathrm{h}=1 \mathrm{~mm}$, the same area corroded width $\Delta \mathrm{L}=4 \mathrm{~mm}$, and depending on different positions of the corroded area.Figures 5,6 and 7 show the variation of the frequency of the first bending mode in the OXY plane, the variation of the bending frequency in the $\mathrm{XXZ}$ plane and the torsional frequency according to the depth of corrosion.

\section{Conclusions}

1. The most sensitive modes in changing frequencies along with the depth of corrosion are bending modes.(Fig 5. Fig 6.)

2. For both bending modes and torsion modes, the most sensitive mode to modify the frequency with the depth of corrosion is the first mode. (Fig.7 and Fig 8.) The first bending mode is more sensitive depending on the corroded depth than the first torsion mode. Percentage is $6.9 \%$ versus $2.23 \%$.

3. This last conclusion is experimentally very favorable because the first bending mode is the simplest to excite by a mechanical impulse.

4. The decoupling of bending vibrations in the two planes as well as torsional vibrations is experimentally useful in the process of signals analysis, because each frequency from spectrum can be assigned to the corresponding vibration type, bending or torsion.

\section{References}

1. G.R. Gillich et al., "A ssessment of Corrosion Damages with Important Loss of M ass and Influences on the Natural Frequencies of Bending Vibration Modes", Applied M echanics and M aterials, V ol. 430, pp. 95-100, (2013)

2. G.R.Gillich, Z. I. Praisach, V. Iancu, H. Furdui and I.Negru, Natural Frequency Changes due to Severe Corrosion in Metallic Structures, Journal of Mechanical Engineering 61(12) pp, 721-730, (2015)

3. H.A. Razak, F.C.Choi, The effect of corrosion on the natural frequency and modal damping of reinforced concrete beams, Engineering Structures, 23(9), pp 1126-1133, (2001)

4. C.Y.Y oung, R.G.Budynas, Roark's Formulas for stress and strain, 7th Edition, M cGraw-Hill Companie, (2002) 